\title{
DISCURSO, LA UNIVERSIDAD DE CHILE Y SU RESPONSABILIDAD NACIONAL
}

Eugenio González Rojas 
Texto íntegro. Eugenio González Rojas, La Universidad de Chile y su responsabilidad nacional, Editorial de la Escuela de Derecho de Valparaíso de la Universidad de Chile, Valparaíso, 1968. 


\section{DISCURSO, LA UNIVERSIDAD DE CHILE Y SU RESPONSABILIDAD NACIONAL}

Desde hace algunos años, es de rutina que al conocerse la selección de ingreso a las Universidades se les formulen acerbas críticas, responsabilizándolas de las frustraciones morales y sociales de los numerosos jóvenes que no obtienen matrícula en las escuelas de enseñanza superior. Estas críticas afectan especialmente a la Universidad de Chile por ser ella, el principal órgano que el Estado tiene para cumplir, en el plano universitario, su deber constitucional de atender, con preferencia, a la función educativa.

Periódicamente, también, sus autoridades dan a conocer la situación académica y financiera en que se encuentra la Universidad de Chile, las exigencias múltiples que su natural crecimiento importa, los planes docentes, científicos y culturales que sería necesario realizar para ajustar su desarrollo orgánico a las demandas -cada vez más variadas, más complejas y más premiosas- de una sociedad como la nuestra, en trance de profundos cambios de su régimen institucional.

Así, en abril de 1966, expusimos, en un extenso documento, entre muchas consideraciones relacionadas con la situación y la perspectiva de la Universidad de Chile, algunas cifras reveladoras de su extraordinario avance, a partir de 1957. Nos parece oportuno reiterarlas, comparándolas con las correspondientes a 1967, para que la opinión pública pueda apreciar la forma en que ella procura cumplir su responsabilidad nacional.

\section{EXPANSIÓN DE LA MATRÍCULA}

La matrícula de nuestra Universidad, que atiende a más del 50\% del alumnado universitario del país, era en 1957 de 12.267 alumnos y alcanzó en 1967 a 30.663. Sólo están incluidos en estas cifras los alumnos regulares que cursan enseñanza superior, es decir, quedan excluidos de ellas los alumnos del Liceo "Manuel de Salas" de la Facultad de Filosofía y Educación, los del Instituto de Estudios Secundarios de la Facultad de Ciencias y Artes Musicales que ascendieron, respectivamente, a 2.565 y 268 , y los alumnos de las Escuelas de Temporada que sumaron 4.602. También los cursos de carácter artesanal que se ofrecen en algunas escuelas. El aumento más significativo se ha producido en los últimos cinco años: en 1962 hubo una matrícula aproximadamente igual a la mitad de la correspondiente a 1967: 15.915 alumnos. Parece superfluo destacar la importancia que este dato tiene para una mejor comprensión de nuestros problemas.

A tan rápida expansión de sus servicios docentes, hay que agregar las nuevas carreras creadas con el propósito de preparar a los muchos profesionales y especialistas, de grado medio, que va requiriendo nuestro desarrollo económico y social. Para apreciar el esfuerzo que en este sentido se ha hecho, baste señalar que en 1957 se daban 66 tipos distintos de formación profesional y que subieron a 116 en 1967, la mayor parte de 
ellos, en los Centros Universitarios de las provincias, cuya eficaz contribución a nuestro adelanto cultural está siendo justamente reconocida.

Resulta evidente, además, que, al preparar en carreras cortas (de cuatro y menos años) profesionales destinados al desempeño de funciones específicas en la educación, la salud, la administración, la agricultura y la industria, se hace posible que los egresados de las carreras largas (de cinco años y más) puedan ser mejor utilizados que ahora por la sociedad y el Estado. Vale tener en cuenta, al respecto, las cifras siguientes: el $76,2 \%$ del alumnado de la Universidad de Chile, en 1957, seguía carreras de cinco y más años de duración y sólo el 23,8\% lo hacía en carreras de cuatro años y menos. Ahora, la proporción es de $52,5 \%$ y $47,5 \%$, respectivamente, previéndose para los años venideros un aumento progresivo del alumnado que prefiere las carreras cortas.

\section{MEJORAMIENTO CUALITATIVO DE LA DOCENCIA}

La considerable expansión cuantitativa de la docencia no ha disminuido su perfeccionamiento cualitativo. Nuestra Universidad revisa periódicamente sus planes y programas de estudio, para adecuarlos a las exigencias del progreso científico y tecnológico y a las realidades y perspectivas del desarrollo nacional. Entre enero de 1966 y mayo de 1967, el Consejo Universitario consideró 46 planes y programas de estudio y reglamentos de Escuelas y de carreras recién creadas.

Dentro del mismo propósito, habría que mencionar el hecho de que nuestra Universidad -con sus propios recursos, a través de convenios con universidades extranjeras y mediante la ayuda de organismos internacionales- ha enviado en los últimos tres años un promedio de 200 miembros de su personal académico a especializarse y perfeccionarse en los más prestigiosos centros científicos y universitarios de Europa y Estados Unidos.

Para mantener a la enseñanza superior en el grado de excelencia que le corresponde, es necesario vitalizarla y enriquecerla con una seria investigación científica y tecnológica. Durante 1966, la Universidad estaba realizando 914 investigaciones en diversas ramas del saber y 1.300 en el año 1967. Las características de nuestro país, hacen, por otra parte, que la asistencia técnica de los organismos universitarios a instituciones públicas y empresas privadas adquiera especial relieve.

\section{EXTENSIÓN UNIVERSITARIA Y ACCIÓN SOCIAL}

Aparte las tareas de enseñanza y de investigación que le son propias, nuestra Universidad es un activo centro difusor de la cultura, que hace llegar los incentivos del saber y del arte y promueve la conciencia de altos valores de vida y de trabajo en todo el territorio de la República. El Departamento de Extensión Universitaria mantiene, en círculos cada vez más amplios de la capital y las provincias, el interés por los grandes problemas humanos, mediante escuelas de temporada, cursos y conferencias. El Departamento de 
Acción Social lleva a apartadas localidades el estímulo de una juventud deseosa de perfeccionar su formación profesional, conociendo directamente las necesidades de nuestro pueblo y ayudándolo a la vez bajo la tuición de investigadores y profesores.

Durante 1967, el Departamento de Extensión Universitaria organizó ocho Escuelas de Temporada, con una matrícula de 4.602 alumnos; 5 cursos y 170 conferencias. Dentro de su Sección de Extensión Estudiantil, funcionaron 5 cursos, con 2.265 alumnos y 82 conferencias de orientación; y, en el Centro de Estudios Sindicales y Cooperativos, 8 cursos y 3 seminarios con 250 y 75 alumnos, respectivamente. El Departamento de Acción Social realizó de invierno y 28 de verano, con la participación de numerosos alumnos universitarios, investigadores y profesores, en diversas comunidades urbanas y rurales.

A la expansión experimentada por las actividades universitarias, en "la enseñanza, el cultivo y la difusión de las ciencias y las artes", no ha correspondido una expansión correlativa de sus recursos humanos, materiales, técnicos y financieros. La planta física de la Universidad, por ejemplo, aunque ha aumentado -y en forma inarmónica, dispersa, para solucionar problemas inmediatos- de $139.668 \mathrm{~m} 2$. en 1957 a $273.819 \mathrm{~m} 2$. en 1967 , es insuficiente para que sus servicios funcionen de manera condigna al grado de su desenvolvimiento. Faltan aulas, bibliotecas, laboratorios, gimnasios. Faltan hogares y casinos estudiantiles, canchas deportivas. Faltan, sobre todo, equipos de trabajo -personal y medios- para atender a las crecientes promociones estudiantiles.

\section{SERVICIOS Y PRESUPUESTOS}

Los organismos administrativos de nuestra Universidad se agrupan en quince grandes reparticiones: la Rectoría, la Secretaría General y las trece Facultades. Componen estas quince reparticiones 322 organismos situados a lo largo de todo el territorio nacional, cuyas denominaciones son: escuelas, institutos, centros, departamentos, oficinas, estaciones, observatorios, etc. Ciento cincuenta y seis tienen presupuesto propio y el resto, ciento sesenta y seis, lo obtienen de los anteriores. De la Rectoría dependen, aparte de servicios centrales de administración, el Departamento de Ciencias de Valparaíso, el Instituto de Estadística, el Instituto de Estudios Internacionales, y los ocho Centros Universitarios de provincias: Arica, Iquique, Antofagasta, La Serena, Talca, Chillán, Temuco y Osorno.

Para atender a tan variadas y tan complejas funciones, nuestra Universidad cuenta con un Presupuesto constituido por sus entradas propias (derechos de matrícula, prestación de servicios, producto de leyes especiales, etc.) y por los aportes anuales del Fisco. Dejando de lado las primeras, que permiten solventar actividades en cierto modo marginales, resulta ilustrativo para la opinión pública conocer en qué medida se ha aumentado, en los últimos tres años, la cuota del Estado para el funcionamiento de su principal institución de enseñanza superior.

Las cifras que vamos a exponer explican sobradamente las dificultades cotidianas que afrontan los servicios en su funcionamiento y las limitadas posibilidades de expansión que ellos tienen para afrontar las perentorias exigencias que el aumento del 
alumnado implica. Sólo daremos cifras significativas: la información completa está a disposición de quien quiera conocerla en el Departamento de Presupuesto y Finanzas de la Casa Central.

\section{INCREMENTO DEL APORTE PRESUPUESTARIO FISCAL}

Las cantidades totales solicitadas como mayor aporte del Estado para mantener y perfeccionar las tareas docentes, científicas y culturales de nuestra Universidad al ritmo exigido por las crecientes demandas de matrícula, el fomento de la investigación científica y tecnológica aplicada al estudio de problemas directamente vinculados al desarrollo de la riqueza pública y a la difusión de la cultura en más amplios círculos sociales fue en 1966, de E: $96.000 .000 \mathrm{~m} / \mathrm{m}$. de los cuales solo se otorgaron $\mathrm{E}^{\circ}$ 14.000.000; en 1967 fue de $\mathrm{E}^{\circ} 80.000 .000 \mathrm{~m} / \mathrm{m}$., de los cuales sólo se otorgaron 17.000.000. Para el año en curso, el mayor aporte solicitado fue de $\mathrm{E}^{\circ} 99.000 .000 \mathrm{~m} / \mathrm{m}$. y le han sido otorgados hasta ahora, $E^{\circ}$ 19.000.000.

Un sucinto análisis de las cifras anteriores permite establecer que, sin considerar los reajustes de remuneraciones que se otorgan cada año por ley especial, y que pasan a incorporarse al presupuesto del año venidero, el mayor aporte fiscal para el presupuesto de gastos corrientes fue en 1996 de un 7,31\% del total de $\mathrm{E}^{\circ}$ 97.164.966, asignado en 1965; en 1967, de un 10,92\% del total de $E^{\circ} 134.071 .000$, asignado en 1966, y en 1968 , de un 9,83\% del total de $\mathrm{E}^{\circ} 174.065 .000$, asignado en 1967. Estos porcentajes incluyen los aumentos de sobresueldos (trienios, cargas familiares) que se producen en el curso de cada año, los incrementos vegetativos de los rubros de bienes de consumo, y los gastos que implican la continuación de cursos iniciados en períodos anteriores.

De este modo, las disponibilidades para extender las actividades internas y externas de la Universidad se reducen hasta proporciones insignificantes. Cabe agregar que en el presupuesto de gastos corrientes, el Fisco dejó de pagar E 2.350 .033 (1.4\%), suma que, por corresponder a compromisos contraídos, deberá cubrirse con el mayor aporte de 1968, el que se rebaja así, del 8,83\% al 7,5\%. Inoficioso sería subrayar la incidencia que ello tendrá aun en el mantenimiento mismo de la actual situación universitaria.

Los aumentos del presupuesto de capital han sido los siguientes: en 1966, un 33\% de los $\mathrm{E}^{\circ} 10.000 .000$ a que ascendió el Presupuesto de 1965; en 1967, un 27,82\% de los $\mathrm{E}^{\circ} 13.300 .000$ a que ascendió el Presupuesto de 1966; y en 1968 de un 20,83\% de los $\mathrm{E}^{\circ} 17.000 .000$ a que ascendió el Presupuesto de 1967. Durante 1967, el Fisco dejó de entregar el 33\% del total del aporte: $\mathrm{E}^{\circ}$ 5.666.666, cantidad íntegramente comprometida en trabajos ya hechos, los cuales deberán pagarse con cargo al presupuesto de capital de 1968, que así se reduce en un 27,55\%.

El aporte fiscal en dólares correspondiente a 1967 -US\$1.090.000- fue inferior en un 18\% al del año 1966. Del presupuesto para el año 1966 -US\$ 1.330.000- el Fisco entregó un $96,7 \%$, dejando de pagar, por lo tanto, US\$43.755, y del presupuesto de 1967 -US\$ 1.090.000- ingresó a la caja de la Universidad solamente el 52,2\%. Es fácil suponer el negativo efecto que tan considerable reducción a la cuota de dólares 
-US\$ 520.595- ha tenido en el funcionamiento de las escuelas cuyas actividades docentes y científicas requieren de material importado.

Muy ilustrativo es, también, el empleo de los fondos provenientes de la ley, 11.575, promulgada en 1954, por la que se destina, en forma porcentual, a las Universidades chilenas, el medio por ciento de todos los tributos fiscales, a fin de favorecer en ellas la investigación científica y tecnológica orientada a mejorar la productividad de la agricultura, la industria y la minería, y a acelerar el desarrollo económico mediante la utilización racional de las riquezas nacionales. Nuestra Universidad debe recibir 10/18 de su rendimiento, exclusivamente para tales fines.

A partir de 1965, sin embargo, debido a que el Erario no pudo financiar en su totalidad las necesidades mínimas de nuestra Universidad, se la ha autorizado en la Ley de Presupuesto de la Nación parta invertir los fondos provenientes de la ley 11.575 en gastos de funcionamiento. Es decir, ingresos que son propios de la Corporación, con específico destino en la ley de origen, están sirviendo para financiar servicios que, sin ellos, tendrían que reducir su actividad en apreciable medida, cuando no suspenderla por completo.

De los $E^{\circ} 7.6000 .000$, estimados como rendimiento de la Ley 11.575 para nuestra Universidad en 1965, sólo pudieron invertirse en investigaciones científicas y tecnológicas $\mathrm{E}^{\circ} 3.635 .447$ (47,83\%) y en 1966, de $\mathrm{E}^{\circ} 12.000 .000$, calculados, sólo $\mathrm{E}^{\circ} 2.257 .969$ $(12,82 \%)$. De los $E^{\circ} 13.300 .000$, presupuestados para 1967, la mayor parte fue destinada también a fines extraños a la ley, pero al cerrarse el ejercicio anual no habían ingresado a la Tesorería General E ${ }^{\circ}$ 9.557.570. Giros recibidos al terminar enero, han reducido la deuda pendiente del Fisco a E ${ }^{\circ}$ 4.853.317. El Fisco adeuda, además, a nuestra Universidad la totalidad del rendimiento en dólares de la Ley 11.575 correspondiente a 1967 -US\$ 456.000- y el 40\% del correspondiente a 1966: US\$ 188.099

\section{PLANIFICACIÓN Y REFORMA}

Frente a la demanda de mayores recursos para nuestra Universidad, se manifiestan, dentro y fuera de ella, opiniones en el sentido de que aquellos de los cuales dispone, distribuidos racionalmente conforme a una planificación técnica de sus actividades, le permitirían mejorar y expandir sus servicios, dándose a entender, así, que en la administración universitaria existiría arbitrismo dispendioso, por falta de una política coherente y bien orientada que fije objetivos, etapas y prioridades en el funcionamiento de la Corporación.

La administración universitaria adolece, sin duda, de muchos de los defectos que parecen ser inherentes a la de cualquier servicio público, dentro de un Estado de acentuada índole burocrática. La complejidad de las disposiciones legales y reglamentarias a que está sometida nuestra Universidad impide, en efecto, cualquier simplificación de sus mecanismos administrativos, cada vez más recargados de enojosos trámites que perturban, a menudo, la oportuna atención de las necesidades propiamente académicas. Consideramos de urgencia dotarla, mediante una nueva ley orgánica, de la potestad reglamentaria requerida para que ella misma regule su régimen interno. 
Antojadiza nos parece en cambio, -cuando menos inconsulta- la suposición de que nuestra Universidad carece de una definida política, reflejada en una planificación técnica de su actividad corporativa, condición esta última del óptimo aprovechamiento de los recursos disponibles y base objetiva para solicitar la entrega de mayores aportes fiscales. La verdad es otra: todas las Facultades están utilizando el sistema de los Presupuestos por programas, que recomiendan los expertos en la materia, y hacen sus peticiones de fondos para realizar proyectos específicos de docencia y de investigación que luego son analizados en su conjunto por las autoridades competentes.

El Consejo Universitario y la Rectoría, asesorados por las Oficinas de Planificación y de Construcciones Universitarias, creadas en 1966, han estudiado y aprobado en sus aspectos fundamentales un vasto plan de expansión de la Universidad, hecho a base de las exigencias técnicamente estimadas del desarrollo educacional y nacional en los próximos diez años. Este plan se revisa constantemente para actualizar sus proyecciones, a medida que institutos de la propia Universidad y otros institutos del Estado van perfeccionando el análisis de las posibilidades, los requerimientos y las tendencias de nuestra evolución colectiva.

Al planificar su acción y su desarrollo, se ha tenido en vista la necesidad de modificar la estructura de los servicios universitarios en el ámbito nacional y el régimen de su funcionamiento interno. Pero ello requiere el pronto reemplazo de nuestro Estatuto Orgánico, en vigencia desde 1931, por otro más flexible que entregue a nuestra Universidad -dentro, naturalmente, de los preceptos de carácter fundamental que corresponde establecer en la ley- una amplia potestad reglamentaria, para que ella pueda darse las normas reguladores de su vida académica y administrativa.

El Consejo Universitario ha discutido y aprobado -recogiendo esta vez las opiniones expuestas en las distintas Facultades- los principales artículos de un nuevo proyecto de Estatuto Orgánico que espera elevar a la consideración y resolución del Gobierno y el Parlamento al iniciarse el próximo año académico. Todas sus disposiciones responden al propósito de que la Corporación disponga de medios legales expeditos para cumplir sus tareas con el máximo de eficacia y sentido de futuro, organizando sus servicios -en Santiago y las provincias- como estime conveniente a sus propios fines y democratizándolos de manera que la "comunidad universitaria" -tan manida actualmente en la retórica académica- sea, con plena autenticidad, una forma de vida y de trabajo.

¿Cómo ha podido nuestra Universidad -actuando en el rígido marco legal de un Estatuto Orgánico inadecuado a sus necesidad de reforma y desarrollo, y, lo que es acaso más grave, perturbada en el cumplimiento de sus deberes de rutina por un presupuesto insuficiente- mantener sin alteración notoria el pulso de su actividad regular y aún acrecentar, cada año, el número de sus alumnos? Sólo mediante un generoso esfuerzo de su personal -tanto el académico como el administrativo- obligado a trabajar, por lo común, en condiciones materiales insatisfactorias y con rentas cuyo monto está lejos de corresponder a la preparación que se le exige y a la responsabilidad que le concierne.

La emigración de profesionales y científicos hacia países ricos en que encuentran atractivas oportunidades, constituye un problema nacional que el Estado habrá de abordar sin tardanza por sus negativos efectos en funciones vitales de servicio público y 
de progreso social; pero es todavía más grave que profesionales y científicos altamente calificados abandonen la Universidad por situaciones mejores -no sólo en cuanto a renta, sino también a medios de trabajo- que les ofrecen instituciones internacionales, empresas privadas y -lo que es inadmisible- otros organismos del Estado o en los cuales el Estado tiene influencia predominante.

¿No será posible, al menos, establecer en la Ley impedimentos que eviten tan absurda competencia entre organismos del llamado "sector público"? De continuar ella, se resentirá progresivamente en su calidad, la formación de los futuros profesionales que, por el contrario, deberán ser, en el futuro, mejor preparados para asimilar los adelantos acelerados de la ciencia y de la técnica y para afrontar con capacidad creadora la solución de los inquietantes problemas de las sociedades modernas, sobre todo de aquellas, que como la nuestra, reclaman un progreso rápido y efectivo en todos los órdenes de su vida.

\section{INSUFICIENCIA DE RECURSOS}

No faltan, pues, planes, sino medios. El Consejo Universitario ha aprobado las ideas centrales de un proyecto de reorganización de nuestra Universidad que, para ser puesto en práctica, requiere construir previamente, en plazo corto, nuevas edificaciones agrupándolas en Centros Docentes que permitan la integración armónica de servicios similares, ahora dispersos. Los aportes de capital, a pesar de sus incrementos anuales que, como se ha visto en 1967 pueden resultar precarios, no permitirían, por sí solos, la realización del plan de nueva planta física, elaborado por la Oficina de Construcciones Universitarias.

De ahí que se haya recurrido a préstamos en el exterior. Aparte los obtenidos para construir edificios destinados a los Centros Universitarios de las provincias, a escuelas de la Facultad de Ciencias Físicas y Matemáticas, a parte de la Escuela de Salubridad y otro menores, el Banco Interamericano de Desarrollo prestó con destino a las Facultades de Agronomía y Medicina Veterinaria, en 1967, la suma de US\$ 5.000.000, con los cuales se construyen en el fundo Antumapu, a corta distancia del centro de la capital, las instalaciones que harán de él, presumiblemente, el conjunto funcional mejor dotado para la enseñanza y la práctica de las disciplinas agropecuarias de Latinoamérica. Actualmente el BID está examinando una solicitud de préstamo por US\$25.000.000, que se aplicarían a la realización del plan de reorganización académica y administrativa de nuestra Universidad y a la ampliación y mejoramiento de su planta física.

Hemos procurado poner de relieve que los mayores aportes fiscales de cada presupuesto en los últimos años apenas alcanzan para que nuestra Universidad cumpla restringidamente sus funciones, con un personal insatisfecho por sus rentas, y en aulas, laboratorios y bibliotecas que hace tiempo dejaron de corresponder, por su cabida y sus dotaciones al número de alumnos admitidos, casi en todas las escuelas superiores al recomendable para impartir una buena enseñanza. Los proyectos de expansión, oportunamente presentados, quedan recargando los archivos de alguna oficina del Estado, 
como muestra y testimonio de la preocupación de las autoridades universitarias acera de los problemas que se les plantean y sus eventuales soluciones.

\section{SITUACIÓN EN EL PRESENTE AÑO}

Para el presente año, la situación universitaria reviste caracteres de acentuada gravedad. Sobre la base de serios estudios hechos por los directores de los servicios y revisados enseguida por los decanos de las Facultades, nuestra Universidad solicitó un mayor aporte total al presupuesto (gastos corrientes, de capital y en dólares) -en el que se incluye, aparte el aumento vegetativo en los rubros de rutina, lo necesario para continuar cursos, aplicar nuevos planes de enseñanza, iniciar programas científicos y ampliar las matrículas de aquellas escuelas en condiciones de hacerlo- de $E^{\circ}$ 99.000.000. Le han sido otorgados hasta ahora -como ya se dijo- $\mathrm{E}^{\circ}$ 13.000.000.

La matrícula en nuestra Universidad ha aumentado entre 1961 y 1967 en los siguientes porcentajes anuales: 7,6\%, 9,7\%, 12\%, 14,5\%, 19,1\% y 14,9\%. Ellos demuestran cómo en forma inequívoca ha crecido rápidamente hasta 1966 y que entre 1966 y 1967 experimentó un descenso. Teniendo a la vista las cuotas fijadas para 1966, el porcentaje de 1967 experimentará un ligero ascenso explicable por la continuación de los cursos creados en años recientes. Esto indica que nuestra Universidad ha llegado al límite de utilización de su personal, de sus instalaciones y de sus locales.

Los datos precedentes tienen, pues, por objeto, que la opinión pública y aquellos jóvenes que este año, como en los anteriores, no han obtenido matrícula en nuestras escuelas, aprecien la magnitud y trascendencia del problema cuyo planteamiento corresponde a los organismos técnicos de nuestra Universidad, pero cuya solución depende de la voluntad política del Estado. No sería patriótico aminorarlo, con sutiles análisis estadísticos, ni menos eludirlo en generalidades teóricas sobre optimistas perspectivas del desarrollo nacional. Hay que afrontarlo.

¿Cómo podrá pedirse a nuestra Universidad la admisión de una cuota mayor de la prevista -alrededor de 10.000 alumnos nuevos- de los 29.800 que se sometieron a las pruebas para ingresar a sus aulas? Es efectivo que muchos de ellos no demostraron reunir las condiciones indispensables para seguir con probabilidades de buen éxito carreras de carácter universitario; pero también es efectivo que entre los rechazados por falta de plazas en las escuelas los hay, en considerable número, que, reuniéndolas en grado a lo menos suficiente, han quedado al margen de inmediatas posibilidades en la educación superior.

Puede afirmarse, sin necesidad de recurrir a los escasos estudios de recursos humanos -todos imprecisos, fragmentarios, y sobremanera discutibles- que hay en el país carencia de profesores, ingenieros, médicos, veterinarios, agrónomos, enfermeras, asistentes sociales y otros profesionales, como asimismo de técnicos de grado intermedio para el desempeño de funciones específicas de colaboración en la industria, la agricultura, la administración, la salud, etc. Conviene a nuestro desarrollo económico-social y normalizaría nuestro sistema educacional la orientación de un número creciente de egresados de la enseñanza media hacia ese tipo de carreras cortas. 


\section{LA DEMOCRATIZACIÓN DE LA UNIVERSIDAD}

Sin embargo, habrá siempre impedimentos insuperables para un acceso masivo a la formación profesional de carácter universitario: por una parte, la capacidad exigida a los postulantes; por otra, la necesidad social. No cualquiera, por el simple hecho de haber terminado el ciclo secundario puede atribuirse el derecho a ingresar a la Universidad: tiene que acreditar, para ello, calidad y vocación. Además, es obvio que ningún Estado, cualquiera sea el régimen imperante en él, contribuiría a formar más profesionales de los requeridos por la sociedad para su subsistencia y progreso.

La democratización de la Universidad -y nos referiremos a este concepto en su alance externo, social- no puede entenderse en tan peregrino sentido, pero es legítimo preguntarse: ¿llegan actualmente a la Universidad los jóvenes más capaces para los estudios superiores? El proceso educativo iproduce, en sus diversas instancias, una selección auténtica y una adecuada distribución de los alumnos, según sus aptitudes y vocaciones? Ni lo uno, ni lo otro. La selección universitaria se realiza entre jóvenes que por pertenecer, en abrumadora mayoría, a grupos sociales de alta y mediana situación económica, pudieron completar el ciclo de enseñanza media. ¿Cuántos niños de talento quedarán, sin ocasión de desplegar sus virtualidades, en las primeras etapas de la educación sistemática?

La democratización de la enseñanza superior sólo será efectiva cuando se democratice realmente la educación nacional en su conjunto y esto sólo será posible cuando se modifiquen las estructuras básicas de la sociedad y del Estado. Por ahora, sólo se puede atenuar la injusticia del actual estado de cosas mediante una próvida asistencia del Estado a los niños y jóvenes de escasos recursos familiares y manifiestas capacidades intelectuales, para que puedan mantenerse dentro del sistema educativo hasta llegar a la Universidad.

También puede entenderse la democratización de nuestra Universidad en sentido distinto. Por su índole de institución estatal, ella debe servir activamente al pueblo que la sustenta. La ciencia y el arte que en ella se cultivan no pueden ser privilegio y regodeo de intelectuales y estetas, apartados de los sobrecogedores problemas de un mundo en crisis, en circunstancia que el destino de la humanidad parece amenazado. De ahí que sea esencial función suya llevar al pueblo estímulos de cultura, a fin de abrir los espíritus a la comprensión de los valores de la verdad, la belleza y la justicia para el logro de una convivencia digna.

\section{ALGUNAS NORMAS UNIVERSITARIAS}

Nuestra Universidad es ajena a las contingencias de la política partidista, pero no puede ser indiferente a los movimientos de progreso social. Por su condición de entidad moral, debe tomar conciencia de las fuerzas creadoras que especialmente en los períodos críticos, emergen como impulsos ciegos de la voluntad colectiva. "La Universidad -dice un acuerdo reciente del Consejo Universitario- está obligada a participar eficazmente 
en el proceso de cambio social, orientando las múltiples formas de su actividad interna y externa en el sentido de sus tendencias más progresivas".

Aplicando este criterio normativo, estamos empeñados en darle a nuestra Universidad una estructura nueva que le permita ser una comunidad de vida y de trabajo, donde la autoridad, la jerarquía y la disciplina se fundamenten en principios, normas y valores libremente aceptados por profesores y alumnos. Los problemas que en ella se planteen deben resolverse en términos de razón y justicia, con leal prescindencia de cualquier propósito de presión sobre el espíritu y la conducta de individuos y grupos. La violencia y el dogmatismo - que es también una forma de violencia- deben ser excluidos del ámbito universitario.

Cuanto se haga por erradicar de la comunidad universitaria el dogmatismo y la violencia le reforzarán en su carácter de tal, facilitando su labor constructiva. Hay que tener presente, sin embargo, una realidad: la rebeldía estudiantil en muchas universidades del mundo -por motivaciones circunstanciales diversas- es sólo una de los tantos fenómenos reveladores de una crisis histórica en la que se están definiendo nuevas formas de valores de la vida humana. Ha surgido tanto en sociedades políticamente libres y prósperas como en sociedades agobiadas por la opresión y la miseria. Curiosamente, los estudiantes europeos y norteamericanos, hasta hace poco tiempo conformistas en su rigurosa tradición, parecen estar adquiriendo la conciencia política y el sentido social que han caracterizado, desde hace décadas, a los de nuestra América latina.

Nos parece altamente plausible que nuestros estudiantes tengan serias inquietudes políticas y sociales. Por lo demás, las han tenido siempre y, a través de su Federación, han actuado como factor dinámico del progreso nacional. Preocupándose de los problemas públicos y promoviendo el cambio social, se han sustraído a las limitaciones espirituales de una formación sin base humanista y demasiado especializada que, por natural consecuencia, pudo inclinarlos a un concepto mezquinamente utilitario de su ejercicio profesional.

\section{LA REPRESENTACIÓN ESTUDIANTIL}

Nos referimos anteriormente a lo que pudiera llamarse la democratización de la Universidad hacia afuera, en su relación con el aumento de las cuotas anuales de matrícula de manera que tengan acceso a sus aulas todos los jóvenes capaces y, en especial, los que provengan de familias de bajos ingresos. Ahora, consideraremos lo que pudiera llamarse la democratización de la Universidad hacia adentro, es decir, con relación al funcionamiento de sus servicios docentes, científicos y culturales y, sobre todo, con el modo de elegir a los miembros del cuerpo académico superior y sus autoridades. Concretamente se ha planteado un problema: ¿deben los estudiantes intervenir en esta elecciones, con derecho a voto, para que le democratización de la Universidad sea efectiva?

Desde hace decenios, los estudiantes de nuestra Universidad participan en diversos organismos, por disposiciones internas de Escuelas y Facultades -generalmente sólo con derecho a voz, en algunos con derecho a voto- y asisten a sus sesiones, por acuerdo del propio Consejo Superior, tres representantes de la Federación de Estudiantes de 
Chile. La colaboración de los estudiantes ha sido útil, inteligente y constructiva y, en los debates del Consejo Universitario, han expuesto bien documentados, con serena y respetuosa firmeza, sus puntos de vista sobre todos los problemas universitarios. Como en el Consejo Superior es infrecuente que se recurra al voto porque siempre se busca el consenso, los estudiantes participan de verdad en los acuerdos que se adoptan.

No obstante, para la debida integración y el cabal funcionamiento de una genuina comunidad universitaria -y recogiendo la ya larga experiencia del carácter positivo de la colaboración estudiantil- el Consejo Superior, en acuerdo con los delegados de la Federación de Estudiantes, ha resuelto que se institucionalice, dándole vigencia legal, en el nuevo estatuto orgánico, la participación de representantes estudiantiles en todos los organismos colegiados de nuestra Universidad, con derecho a voz y voto desde la cátedra, concebida como grupo de trabajo, hasta el Consejo Superior.

También la tendrán representantes del personal agregado a la docencia. El derecho a voto de los estudiantes no regirá para la elección de autoridades y profesores y la resolución de problemas administrativos.

Este acuerdo ejemplar revela la madurez y seriedad de los dirigentes estudiantiles, a la vez que el espíritu realista y renovador de los consejeros universitarios. Bien han comprendido los estudiantes que en la elección de profesores y autoridades no se trata de configurar en la Universidad "estructuras de poder", asimilables a las que se dan en el Estado, sino de apreciar calidades intelectuales y éticas en función de un orden muy distinto de valores que, en el caso, son los del espíritu y la cultura. Confundir ambas situaciones, puede tener consecuencias extremadamente nocivas en el futuro de la institución universitaria.

\section{CARRERA ACADÉMICA}

Lo dicho no significa que desconozcamos las muchas deficiencias que existen en la generación del cuerpo académico y, por lo mismo, en la generación de las autoridades universitarias. Actualmente, sólo participan en las elecciones universitarias "los profesores ordinarios, los profesores extraordinarios que hayan cumplido cinco años no interrumpidos de enseñanza como tales en la Universidad y estén impartiendo dicha enseñanza, los contratados y los miembros académicos de las Facultades. Cuando se trata del nombramiento de profesores ordinarios sólo tendrán derecho a voto los miembros docentes de la Facultad" (art. 47 del estatuto universitario en vigencia).

La gran mayoría de los claustros electores -los de las Facultades y el Claustro Pleno- están constituidos por los profesores ordinarios, que lo son por desempeñar horas de clases de las llamadas universitarias. Algunos lo son por un muy reducido número de horas de docencia. No participan, en cambios, en los claustros electores, las demás categorías de profesores y los investigadores, aunque gran número de ellos sólo trabajan en la Universidad, afectos a los regímenes de rentas globales, jornada completa y dedicación exclusiva, y reúnen los requisitos docentes y científicos para ser considerados en el más alto rango de la jerarquía universitaria. 
Urge poner término a esta situación evidentemente anómala, estableciendo una "carrera académica" en la que los miembros del personal docente y científico, independientemente de la función que desempeñen, vayan ascendiendo en los diversos rangos de la jerarquía universitaria, hasta alcanzar el más alto que les dará acceso, con plenitud de derechos, a los Claustros de las Facultades y al Claustro Pleno. El respectivo reglamento deberá ser extremadamente riguroso, a fin de que las promociones se produzcan exclusivamente a base de méritos, experiencias y trabajos.

Estamos ciertos de que, a través de la carrera académica, la jerarquía universitaria llegará a ser intrínsecamente legítima, se evitará cualquiera injusta arbitrariedad de las autoridades en el nombramiento del personal docente y científico que sea de su incumbencia y no quedará librado a mayorías ocasionales de las Facultades, a veces movidas por intereses ajenos a los propios de la Universidad, la elección de los catedráticos: sólo podrán serlo quienes estén, previamente, en el grado más alto de la jerarquía universitaria.

\section{LIBERTAD ACADÉMICA}

La libertad académica -lo hemos reiterado recientemente- es principio esencial en la vida y el trabajo de una verdadera Universidad. Para su cabal ejercicio es condición básica, por una parte, la autonomía de la Universidad, con respeto a poderes externos, en el cumplimiento de sus fines docentes, científicos y culturales; por otra, en el orden interno de la Universidad, la libertad académica implica completa independencia de sus miembros para exponer y sustentar ideas y doctrinas. Ningún miembro de la Universidad puede ser objeto de medidas restrictivas, menos de sanciones directas o indirectas por las ideas y doctrinas que exponga y sustente, tanto en los organismos universitarios y ante sus alumnos dentro y fuera de las aulas, como en cualquiera forma de la actividad pública.

Si bien es cierto que nuestra Universidad, por su naturaleza de institución nacional y órgano del Estado, debe mantenerse al margen de los intereses transitorios de la política partidista, ello no puede significar que permanezca ajena a las inquietudes ideológicas y a los apremiantes problemas de nuestra época. Por el contrario, debe estar abierta a la discusión elevada de los grandes temas del conocimiento y de la vida y al examen objetivo de todas las ideas y doctrinas, hecho con la libertad de crítica y el decoro intelectual, propios del espíritu científico. Aún más: la Universidad, como entidad moral, tiene la obligación de promover e la juventud de sus aulas una clara conciencia de los valores que orientan, en sentido de progreso, la formación del hombre y el desarrollo de la sociedad.

Corresponde en primer término, a las autoridades universitarias respetar y hacer respetar el principio de la libertad académica. Ninguna decisión administrativa relacionada con actuaciones de profesores o estudiantes que pudiera aparecer vulnerando el principio de la libertad académica, debe ser adoptada sin un previo, imparcial y exhaustivo estudio de los antecedentes, y audiencia de los afectados, por una comisión 
especial. El nuevo Estatuto Orgánico dispondrá lo que sea pertinente para que no sufra mengua alguna, en tales casos, el interés de la justicia y quede garantizado, en plenitud, el derecho de los miembros de la comunidad universitaria.

\section{AUTONOMÍA Y PLANIFICACIÓN}

No hay, ni puede haber -a nuestro juicio- oposición entre el ordenamiento de las actividades nacionales - en lo que atañe, por supuesto, a la vida material de la sociedad-y la autonomía de la Universidad. El crecimiento de la enseñanza superior se ha estado realizando al azar de iniciativas que traducen los intereses de grupos diversos y es legítimo preguntarse si ella corresponde siempre a nuestras necesidades y si se imparte en el grado de excelencia que su función social requiere. Nuestra Universidad ha expresado en varias oportunidades su pensamiento acerca de la urgencia de planificar su pensamiento acerca de la urgencia de planificar el desarrollo de la enseñanza superior, vinculándola al desarrollo de las demás ramas del sistema educativo y al desarrollo cultural, social y económico de la Nación.

Para avanzar en esta impostergable tarea, consideramos necesario que se establezca en una ley, como organismo del Estado, el Consejo de Coordinación y Planeamiento de la Educación Superior, creado por decreto del Ministerio de Educación en 1967. Le correspondería entrar de inmediato al examen de los proyectos de expansión de las actividades universitarias estatales y particulares, para que en el presupuesto de 1969 se contemplen los correspondientes fondos, distribuidos de manera racional y conforme al interés público. Nos parece incuestionable que la prioridad, en el financiamiento de nuevos servicios y proyectos -carreras, investigaciones, etc.- deben tenerla las Universidades del Estado, en particular la nuestra, que atiende a más de la mitad del alumnado universitario de Chile.

Muchos y difíciles problemas -todos ellos de trascendencia en el desarrollo nacional- se presentan simultáneamente a la consideración del Gobierno y del Parlamento. Entre ellos, el adecuado financiamiento de nuestra Universidad. Los actuales ingresos del Estado, aunque fueran óptimamente distribuidos con inflexible austeridad, acaso sean insuficientes para darles, en plazo breve, solución eficaz. Pero de tal modo está ligada la acción de nuestra Universidad a la vida y el porvenir del país, a través de las generaciones formadas en sus aulas, que estamos seguros de encontrar en el Gobierno y el Parlamento, no sólo comprensión de nuestras necesidades sino, sobre todo, la voluntad de proporcionar los recursos para satisfacerlas. 